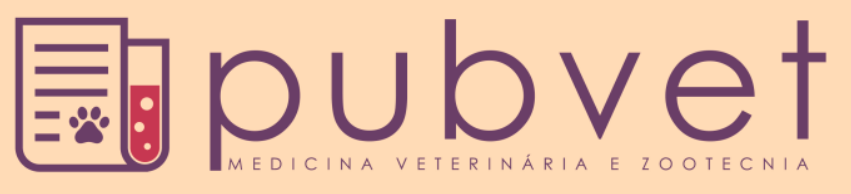

https://doi.org/10.22256/pubvet.v12n2a40.1-4

\title{
Atendimento clínico veterinário em cães e gatos na comunidade Serra Nova do município de Bom Jesus-PI
}

\author{
Sávio Matheus Reis de Carvalho ${ }^{0}$, Wliglesio Leite de Sousa ${ }^{\bullet}$, Alline Alves \\ Rodrigues $^{\bullet}$, Mariane Vieira de Sá ${ }^{\bullet}$, Isael de Sousa Sá ${ }^{\bullet}$, Raffaella Maria de \\ Oliveira Batista ${ }^{1}$, Douglas dos Santos Rocha ${ }^{\bullet}$, Joisiane da Silva Santos ${ }^{\bullet}$, Lygia \\ Silva Galeno ${ }^{2}$, Antônio Augusto Nascimento Machado Júnior ${ }^{3}$
}

${ }^{1}$ Discente em Universidade Federal do Piauí, Campus Profa. Cinobelina Elvas. Bom Jesus - PI. Brasil. E-mail: savioreis1995@gmail.com,

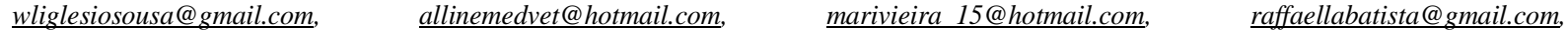
douglasdos santosrocha@hotmail.com, silvajoisiane@gmail.com.

${ }^{2}$ Discente em Universidade Estadual do Maranhão, Campus Paulo VI. São Luís-MA. Brasil. E-mail: lygiagaleno@outlook.com

${ }^{3}$ Docente da Universidade Federal do Piauí, Campus Profa. Cinobelina Elvas, Curso de Medicina Veterinária, Bom Jesus-PI. Brasil. E-mail: marciapbo@gmail.com, machadojunior@gmail.com

*Autor para correspondência, savioreis1995@gmail.com

\begin{abstract}
RESUMO. Desde os primórdios da humanidade, os seres humanos mantêm uma estreita relação com os animais, com o decorrer do tempo, esses animais vêm se tornando como membros da família, necessitando, desse modo, de cuidados mais específicos com relação a sua saúde. O projeto de extensão Pet saudável teve como objetivo levar atendimento clínico, na área de Clínica Médica de pequenos animais, para as comunidades mais necessitadas do município de Bom Jesus- PI. Foram realizadas visitas as comunidades para realizar consultas, coletar material para exames e fornecer orientações sobre a promoção da saúde dos animais, além disso foram confeccionados banners e folders que foram expostos em praça pública e feiras livres, e neles continham informações importantes sobre a criação de cães e gatos. A população atendida pelos alunos mostrou-se satisfeitas com os atendimentos, além disso, o projeto foi importante para os alunos envolvidos, pois através dele possibilitou uma interação da teoria aprendida no decorrer do curso com a prática exercida nos atendimentos. Conclui-se que o projeto de extensão é de grande importância tanto para comunidade acadêmica quanto para a população. No diz respeito aos estudantes pode-se conciliar a teoria com a prática, além de obtenção de experiência principalmente na área de clínica médica de cães e gatos, contribuindo assim para seu futuro profissional. Quanto a população atendida ouve orientações e esclarecimentos no diz respeito a guarda responsável, vacinação, alimentação e cuidados gerais com os animais domésticos.
\end{abstract}

Palavras chave: Animal, comunidades, humanos, município, projeto de extensão

\section{Veterinary clinical care in dogs and cats in the Serra Nova community in the municipality of Bom Jesus-PI}

\begin{abstract}
Since the dawn of mankind, humans have been closely associated with animals, over time, these animals have become members of the family, necessitating more specific health care. The Healthy Pet Extension Project aimed to bring clinical care in the area of Small Animal Clinic to the most needy communities in the municipality of Bom Jesus-PI. Visits were made to the communities to conduct consultations, to collect material for examinations and to provide guidelines on the promotion of animal health. In addition, banners and folders were made in public squares and free fairs and contained important information on the creation of dogs and cats. The population served by the students was satisfied with the attendance; in addition, the project was important for the students
\end{abstract}


involved, because through it enabled an interaction of the theory learned in the course of the course with the practice exercised in the care. It is concluded that the extension project is of great importance for both the academic community and the population. With regard to students, it is possible to reconcile theory with practice, as well as obtaining experience mainly in the area of medical practice of dogs and cats, thus contributing to their professional future. As far as the population served hears guidelines and clarifications regarding responsible care, vaccination, food and general care with domestic animals.

Keywords: Animal, communities, human, municipality, extension Project

\section{Atención clínica veterinaria en perros y gatos en la comunidad Serra Nova del municipio de Bom Jesús-PI}

RESUMEN. Desde los primordios de la humanidad, los seres humanos mantienen una estrecha relación con los animales e con el transcurso del tiempo, estos animales se están convirtiendo en miembros de la familia, necesitando de ese modo de cuidados más específicos con respecto a su salud. El proyecto de extensión Pet sano tuvo como objetivo llevar atención clínica, en el área de Médica de pequeños animales, a las comunidades más necesitadas del municipio de Bom Jesús-PI. Se realizaron visitas a las comunidades para realizar consultas, recoger material para exámenes y proporcionar orientaciones sobre la promoción de la salud de los animales, además fueron confeccionados banners y folders que fueron expuestos en plaza pública y ferias libres, y en ellos contenían informaciones importantes sobre la cría de perros y gatos. La población atendida por los alumnos se mostró satisfecha con las atenciones, además, el proyecto fue importante para los alumnos involucrados, pues a través de él posibilitó una interacción de la teoría aprendida durante el curso con la práctica ejercida en las atenciones. Se concluye que el proyecto de extensión es de gran importancia tanto para la comunidad académica como para la población. En lo que se refiere a los estudiantes se pudo conciliar la teoría con la práctica, además de obtener experiencia principalmente en el área de clínica médica de perros y gatos, contribuyendo así a su futuro profesional. En cuanto a la población atendida hubo orientaciones y aclaraciones en cuanto a la guardia responsable, vacunación, alimentación y cuidados generales con los animales domésticos.

Palabras clave: Animal, comunidades, humanos, municipio, proyecto de extensión

\section{Introdução}

Desde o início da humanidade, os seres humanos mantêm uma estreita relação com os animais. Com o decorrer do tempo, esses animais vêm se tornando como membros da família, necessitando, desse modo, de cuidados mais específicos com relação a sua saúde (Quinton, 2005, ABINPET, 2016).

A procura por serviços médicos veterinários vem aumentando consideravelmente e por esse motivo os proprietários querem proporcionar cada vez mais uma melhor qualidade de vida aos seus animais. Nesse contexto, o Médico Veterinário tem responsabilidade não só de tratar doenças, mas também de orientar e acompanhar o calendário de vacinas e vermifugações, alimentação e indicar a melhor forma de criação dos animais e tudo que envolve as necessidades individuais e particulares de cada espécie, raça e idade (Ferreira et al., 2013). O mercado PET vem aumentando dia mais, e com isso há uma maior necessidade de termos bons profissionais na área. Uma das formas que proporciona o contato do graduando com os animais e tutores é por projetos de extensão (Matos et al., 2012).

Os graduandos têm no projeto de extensão a oportunidade de iniciar o contato com a comunidade e começar a lidar diretamente com os proprietários de animais. Além de levar o aluno a desenvolver um raciocínio para solucionar problemas comuns no dia a dia da prática profissional (Budziak et al., 2017).

O projeto de extensão PET saudável teve como objetivo levar atendimento clínico aos animais de populações carentes do município de Bom JesusPI, como também orientá-los a importância de ter uma posse responsável dos seus animais de companhia. Além disso, possibilitar aos alunos do 
curso de medicina veterinária uma interação entre a teoria e a prática, tornando-os preparados para enfrentar o mercado de trabalho.

\section{Material e Métodos}

Os atendimentos foram realizados pelos alunos do curso de medicina veterinária integrantes do projeto de extensão pet saudável, orientados pelo professor responsável. $\mathrm{O}$ foco dos atendimentos foi o município de Bom Jesus-PI, no bairro Serra Nova. O projeto foi executado seguindo três etapas mensais, sendo a primeira etapa a efetivação da visita ao bairro para o cadastro prévio dos animais que foram atendidos; na segunda etapa foram realizados os atendimentos (Figura 1), coleta de material para exames e prescrição dos tratamentos quando imediatamente possíveis; por fim a terceira etapa onde equipe retornou ao bairro com os resultados dos exames realizados, prescrição dos tratamentos e acompanhamento dos tratamentos já iniciados.

Para orientação da população foram confeccionados banners e folders (Figura 2), expondo-os em praça pública e feiras livres, neles continham informações importantes para a criação de cães e gatos, abordando assuntos como: a importância de alimentar seu animal com ração; a necessidade de vacinar seus animais ainda filhotes; quais os riscos que o animal corre tendo contato com carrapatos; e a necessidade de castrar e os benefícios que a castração oferece, entre outros assuntos que envolvem o bem estar animal e a promoção à saúde.

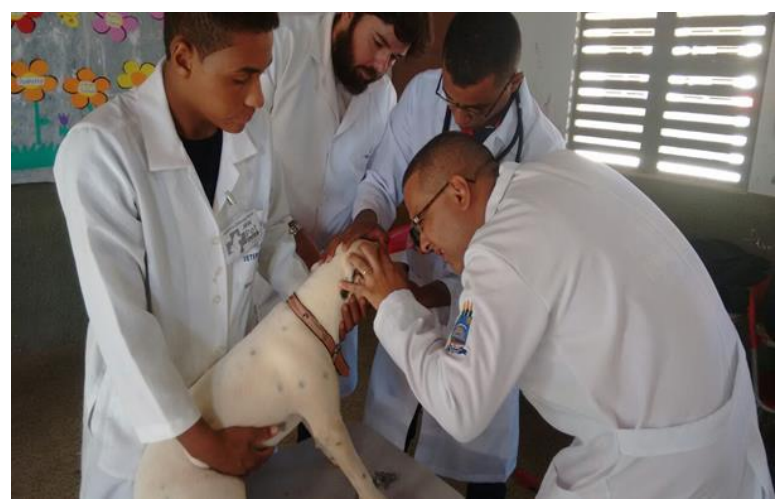

Figura 1. Avaliação clínica semiológica, animal com suspeita de ceratite.

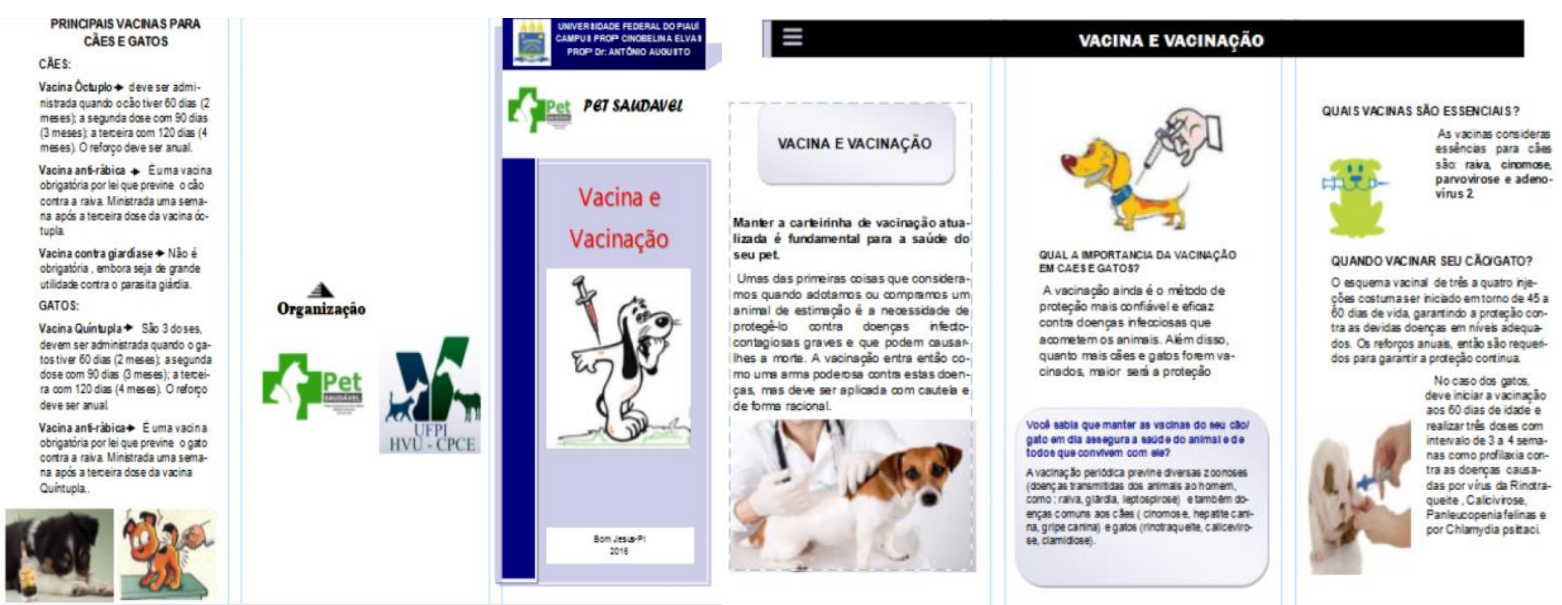

Figura 2. Folder educativo elaborado pelos acadêmicos, contendo informações sobre vacinas e vacinação, e distribuído durante atividades do projeto.

\section{Resultados e Discussão}

A população atendida pelos alunos mostrou-se satisfeitas com os atendimentos, recebendo bem os acadêmicos nas visitas cadastrais, além de levar os animais para o atendimento no dia marcado assim como foram orientados. Para os proprietários e animais que participaram do programa de forma infundada e qualificada os benefícios foram extremamente importantes, pois muitos afirmaram não terem condições de levar seu animal para consulta de rotina ou diagnosticas.

O projeto foi também importante para os alunos envolvidos, pois possibilitou uma interação da teoria aprendida no decorrer do curso com a prática exercida nos atendimentos, que foram oferecidos tais como: os exames clínicos e físicos dos animais; coleta de material para realização dos exames; acompanhamento da carteirinha de vacinação dos animais e prescrição de vacinas e vermífugos. À medida que foram feitos os atendimentos foi possível observar e aprender sobre diversas patologias, como afecções oculares e dermatológicas.

O ensino superior é responsável pela formação do aluno em todas as áreas do conhecimento, e é durante os anos de graduação, nas aulas práticas e 
teóricas, com participação em projetos de pesquisa, elaboração de trabalhos monográficos, estudos individuais e em grupos que se constroem bons profissionais (Camilo \& Ribas, 2007). A conquista da interdisciplinaridade entre ensino, pesquisa e extensão representa hoje um dos maiores desafios das universidades brasileiras (Silva \& Vasconcelos, 2006).

O fluxo, que estabelece a troca de saberes sistematizado, acadêmico e popular, tem como consequências a produção do conhecimento resultante do confronto com a realidade brasileira e regional, a democratização do conhecimento acadêmico e a participação efetiva da comunidade na atuação da universidade. Além de instrumentalizadora deste processo dialético de teoria/prática, a extensão é um trabalho interdisciplinar que favorece a visão integrada do social.

\section{Conclusão}

O projeto de extensão é de grande importância tanto para comunidade acadêmica quanto para a população. No diz respeito aos estudantes pode-se conciliar a teoria com a prática, além de obtenção de experiência principalmente na área de clínica médica de cães e gatos, contribuindo assim para seu futuro profissional. Quanto a população atendida ouve orientações e esclarecimentos no diz respeito a guarda responsável, vacinação, alimentação, entre outros.

\section{Referências Bibliográficas}

ABINPET. 2016. Caderno especial AbinpetAssociação Brasileira da Indústria de Produtos para Animais de Estimação. Agroanalysis, 35, 35-40.

Budziak, C., Pimpão, C. T., Montoya, I. K., Júnior, J. A. V. \& Moraes, P. F. 2017. A importância do projeto Campanha de Castração na formação do profi ssional médico veterinário. Revista Acadêmica: Ciência Animal, 8, 361-370.

Camilo, M. \& Ribas, K. M. F. 2007. Formação docente: professor universitário na atualidade. Revista Eletrônica Lato Sensu, 2, 1-21.

Ferreira, F. P., Dias, R. C. F., Martins, T. A., Constantino, C., Pasquali, A. K. S., Vidotto, O., Freire, R. L. \& Navarro, I. T. 2013. Frequência de parasitas gastrointestinais em cães e gatos do município de Londrina, PR, com enfoque em saúde pública. Semina: Ciências Agrárias, 34, 3851-3858.

Matos, L. V. S., Teixeira, W. F. P., Aquino, M., Viol, M. \& Bresciani, K. D. S. 2012. Orientação sobre posse responsável em uma área endêmica para Leishmaniose Visceral Canina. Revista Ciência em Extensão, 8, 34-41.

Quinton, J.-F. 2005. Novos Animais de EstimaçãoPequenos Mamíferos. Editora Roca, São Paulo.

Silva, M. S. \& Vasconcelos, S. D. 2006. Extensão universitária e formação profissional: avaliação da experiência das Ciências Biológicas na Universidade Federal de Pernambuco. Estudos em Avaliação Educacional, 17, 119-136.

Article History:

Received 16 October 2017

Accepted 19 November 2017

Available online 27January 2018

License information: This is an open-access article distributed under the terms of the Creative Commons Attribution License 4.0, which permits unrestricted use, distribution, and reproduction in any medium, provided the original work is properly cited. 